\title{
Tumor suppressive effect of scavenger receptor class A member 5 overexpression in colorectal cancer by regulating $\mathrm{PI3K} / \mathrm{AKT} / \mathrm{mTOR}$ pathway
}

\author{
Yi Li ${ }^{1}$. Feng Peng ${ }^{1} \cdot$ Xiangyun Tan ${ }^{1} \cdot$ Jin Wang ${ }^{1} \cdot$ Yeqing $\mathrm{Xu}^{1}$
}

Received: 13 March 2021 / Accepted: 13 July 2021 / Published online: 21 August 2021

(c) The Author(s) 2021

\begin{abstract}
Background Colorectal cancer (CRC) exhibits high risks of morbidity and mortality. Objective To investigate the effect of scavenger receptor class A member 5 (SCRAR5) on CRC and its mechanism on modulation of cancer development.

Methods The SCRAR5 expression in four kinds of CRC cell lines (SW620, SW480, HT29, and HCT116) was measured by quantitative PCR and western blotting, respectively. The effects of SCRAR5 abnormal expression on cell proliferation, apoptosis, and migration were analyzed by CCK-8 assay, EdU assay, colony-forming assay, flow cytometry assay, Transwell assay and wound healing assay, respectively. Meanwhile, the involvements of PI3K/AKT/mTOR pathway with the role of SCRAR5 were investigated by western blotting. Afterwards, the in vivo effects of SCRAR5 abnormal expression on CRC xenograft mice were finally investigated by evaluating tumor volume, apoptosis and Ki67 expression.

Results SCRAR5 was lowly expressed in CRC cell lines, especially SW480 cells. Up-regulation of SCRAR5 significantly promoted cell apoptosis, reduced cell proliferation and migration in SW480 cells. Notably, SCRAR5 overexpression obviously inhibited the phosphorylation levels of PI3K, AKT, and mTOR. Reversely, SCRAR5 silence exhibited promoting effects on HT29 cells. Consistently, in vivo experiments also revealed that SCRAR5 overexpression remarkably suppressed tumor volume and Ki67 expression, as well as promoted cell apoptosis.

Conclusions Overall, up-regulating of SCRAR5 obviously inhibited CRC tumor growth in vitro and in vivo, which might be related to PI3K/AKT/mTOR pathway.
\end{abstract}

Keywords Scavenger receptor class A member $5 \cdot$ Colorectal cancer $\cdot$ PI3K/AKT/mTOR pathway $\cdot$ CRC xenograft mice

\section{Introduction}

As the common digestive system malignant tumors, and colorectal cancer (CRC) exhibits high risks of morbidity and mortality (Keum and Giovannucci 2019; Siegel et al. 2020). Currently, the conventional treatment methods for CRC contain chemotherapy, surgical resection, and personalized pharmacological treatments (Mármol et al. 2017; Rejhová et al. 2018). Although the diagnosis and treatments for CRC have the intensive progress, failed chemotherapy

Yeqing Xu

xuyeqing2012@163.com

1 Department of General Surgery, The Affiliated Zhuzhou Hospital Xiangya Medical College CSU, No. 116, South Changjiang Road, Tianyuan District, Zhuzhou 412007, Hunan, China due to drug resistance to chemotherapeutic drugs, higher metastasis and complex pathogenesis result in unsatisfactory prognosis (Mármol et al. 2017). Therefore, the underlying pathogenesis should be investigated to uncover novel and effective treatment targets for CRC.

Over the last few decades, increasing researchers investigate the prognosis biomarkers in order to search for the effective treatment methods for cancers (Shukla 2017). The research and development of antitumor drug targeting prognosis biomarkers has been focused for treating cancer (Wang et al. 2019). Scavenger receptors (SRs) as a cluster of heterogeneous molecules present at the phagocytes surface bind to various ligands such as lipids, proteins, polysaccharides, polyribonucleotides, and modified lipoproteins, thereby enhancing the elimination of harmful non-self or altered-self targets (Gusev et al. 2020). Previous study has demonstrated that adhesion, endocytosis, phagocytosis and signal transduction 
are involved in the clearance of harmful substances (Canton et al. 2013). SRs can be divided into different classes, including scavenger receptor class A member 1 (SCRAR1), SCRAR2 (also named as macrophage receptor with collagenous domain), SCRAR3, SCRAR4, SCRAR5, according to the diverse structure and biological function (PrabhuDas et al. 2017). Notably, SCRAR5 is a newly identified SR that similar to the SCRAR1 and SCRAR2 in the amino acid sequence; however, they exhibit different ligand binding activities (Whelan et al. 2012). SCARA5 is able to deliver non-transferrin bound iron to the kidney which ensures the development of the kidneys, as well as involves with adipogenesis and connective tissue homeostasis (Whelan et al. 2012; Yu et al. 2020a). SCRAR5 is widely expressed in ovary, lung, skin, adrenal gland, kidney, bladder, and testis (Plüddemann et al. 2007). SCARA5 is coded by gene locating on chromosome 8p21 which usually disappears in cancers (Yan et al. 2012). Accumulated studies have demonstrated the abnormal expression of SCARA5 in several cancers, including lung Adenocarcinoma (Yu et al. 2020b), breast cancer (Mamoor 2020), hepatocellular carcinoma (Liu et al. 2018a), and oral squamous cell carcinoma (Liu et al. 2018c). Interestingly, recent study based on bioinformatics analysis has shown that compared with normal tissues, SCARA5 is lowly expressed in CRC clinical specimens (Liu et al. 2020). However, little research has investigated the role mechanism of SCARA5 in CRC.

In the current research, the SCARA5 expression were firstly detected in various CRC cell lines. Then, the effects of SCARA5 abnormal expression on cell proliferation, apoptosis and migration in CRC cells and CRC xenograft mice were unraveled. It is well-known that the mammalian target of rapamycin (mTOR) and phosphatidylinositol-3-kinase (PI3K)/Akt pathways are considered as crucial pathways to regulate cell survival both in pathological and physiological conditions (Ersahin et al. 2015). Accumulating evidence has demonstrated that targeting $\mathrm{PI} 3 \mathrm{~K} / \mathrm{Akt} / \mathrm{mTOR}$ pathway is a potential therapeutic strategy for cancers (Polivka Jr and Janku 2014; Porta et al. 2014). Thus, we explored whether $\mathrm{PI} 3 \mathrm{~K} / \mathrm{Akt} / \mathrm{mTOR}$ pathway participates in the effects of SCARA5 on CRC.

\section{Materials and methods}

\section{Cell culture}

Human normal colonic epithelial cell line HCoEpic and four CRC cell lines, including SW620, SW480, HT29, and HCT116, were purchased from Nanjing KeyGen Biotech. All the cell lines were stored in liquid nitrogen and recovered in complete DMEM medium (Thermo, China) before use.
Then, these cells were cultured in humidified chamber with $5 \%$ carbon dioxide at $37^{\circ} \mathrm{C}$.

\section{Animal experiments}

This study was approved by Experimental Animal Welfare Ethics Committee of Central South University before experiments. Healthy nude mice (weighted 18-22 g) were obtained and randomly assigned to five groups. Mice $(n=5$, each group) were treated with PBS (control group), OE-SCRAR5, OE-NC, shSCRAR5, and shNC via rapid tail vein injection. Then, to obtain the mouse xenograft model, $1 \times 10^{6}$ of SW480 cells per mouse were subcutaneously inoculated in mice. The tumor volume was measured every 1 weeks for 4 weeks. At fourth weeks, the tumor from mice was obtained for the following experiments.

\section{Cell transfection}

For analysis of the impacts of SCRAR5 abnormal expression on CRC cells, SCRAR5 overexpression plasmid (OESCRAR5), SCRAR5 silence plasmid (shSCRAR5), and the corresponding negative controls OE-NC, and shNC were obtained from GenePharma (Shanghai, China). The SW480 or HT29 cells were transiently transfected with these plasmids, respectively, using the Lipofectamine ${ }^{\mathrm{TM}} 3000$ transfection reagent (Thermo).

\section{Cell viability}

The viabilities of SW480 and HT29 cells that underwent the above treatments were evaluated by cell counting kit-8 (CCK-8, Beyotime). Briefly, SW480 or HT29 cells were inoculated to the 96-well plates, and underwent the above treatments for another 24,48 or $72 \mathrm{~h}$, which were then reacted with CCK- 8 at room temperature for $2 \mathrm{~h}$. The absorbances at $450 \mathrm{~nm}$ (OD450) were finally detected by the micro-plate reader. At least three biological replicates of the CCK-8 assay were performed for evaluating SW480 and HT29 cell viability.

\section{5-Ethynyl-2'-deoxyuridine (EdU) assay}

EdU kit (Invitrogen) was utilized to detect cell proliferation. Specifically, the SW480 and HT29 cells at the logarithmic growth phase underwent the above treatments for $48 \mathrm{~h}$, and then cultured with EdU for $2 \mathrm{~h}$. Next, $4 \%$ paraformaldehyde was used to fix cells, and cells were stained with Apollo and 4',6-diamidino-2-phenylindole (DAPI), in turn, and mounted in glass slide. Lastly, the cells were observed using inverted microscope (Olympus, Japan). 


\section{Colony-forming assay}

Addition to EdU assay and CCK-8 assay, colony-forming assay was also utilized to evaluate cell proliferation. In brief, 400 cells per well of SW480 and HT29 cells were seeded in 6-well plates, followed by transfected with OE-SCRAR5, OE-NC, shSCRAR5, and shNC, respectively. After 14 days, cells underwent fixation and incubated with crystal violet. Lastly, the colonies were observed using light microscope (Olympus, Japan).

\section{Cell apoptosis assay}

Annexin V-fluorescein isothiocyanate (FITC)/propidium iodide (PI) kit was used to observe cell apoptosis. The SW480 and HT29 cells were exposed the different treatments for $24 \mathrm{~h}$ and harvested by trypsin. After rinsed with PBS, cells were resuspended with buffer, followed by the exposure of Annexin V-FITC and PI. The treated cells were observed using flow cytometer (BD).

\section{Cell migration assay}

Cell in vitro migration was evaluated using wound healing and transwell assay. For wound healing assay, SW480 and HT29 cells were seeded in 6-well plates and cultured until $60 \%$ of cell confluence. Subsequently, cells were scratched by pipette tips as a vertical lineation, and cultured in serumfree DMEM. Next, cells were transfected with OE-SCRAR5, OE-NC, shSCRAR5, and shNC, respectively. The wound area was measured at 0,24 and $48 \mathrm{~h}$ by light microscope. For the transwell assay, the bottom compartment was added with complete culture medium. The transfected cells were inoculated in the Matrigel Matrix coated compartment with culture medium (serum deprivation) for $24 \mathrm{~h}$. Followed by the cells fixation and staining with crystal violet, the migrated cells were observed by inverted microscope (Olympus, Japan).

\section{Quantitative RT-PCR}

Total RNA samples were obtained from the transfected SW480 and HT29 cells by the Invitrogen TRIzol Reagent (Thermo) following the manufacturer's instructions. Then, complementary DNA (cDNA) samples were prepared from the isolated RNA using the QuantiTect Reverse Transcription Kit (QIAGEN). The following quantitative PCR method was finished using the SYBR Premix Ex Taq TM II (Takara). GAPDH was used as the internal standards for mRNA quantitation. Sequences of primers were all presented in Table 1.
Table 1 Specific primers for qPCR assay

\begin{tabular}{ll}
\hline Gene & Primer sequence \\
\hline SCARA5 & $\begin{array}{l}\text { Sense primer: 5'-TGTGGGCATCTTCATCTTAGC-3' } \\
\text { Antisense primer: 5'-CTCTCATTCAGCCGGTTCAC-3' }\end{array}$ \\
GAPDH & $\begin{array}{l}\text { Sense primer: 5'-GTGGATCAGCAAGCAGGAGT-3' } \\
\text { Antisense primer: 5'-AAAGCCATGCCAATCTCATC-3' }\end{array}$ \\
\hline
\end{tabular}

\section{Western blotting}

The transfected cells were lysed using lysis buffer (Beyotime, China) on ice to extract proteins. Then, proteins samples underwent resolving on PAGE gel and transferring to PVDF, followed by blocking the membrane and reacting with SCRAR5, phosphatidylinositol-3-hydroxykinase (PI3K), phosphorylated-PI3K (p-PI3K), AKT, p-AKT, mTOR, p-mTOR, or GAPDH primary antibody (1:1000, Abcam) at room temperature for $3 \mathrm{~h}$. Second antibody (1:3000, Abcam) was then reacted with the membrane, and the expression of these proteins were observed using enhanced chemiluminescence (ECL, Millipore, USA).

\section{Tunel assay}

Tumor tissues from different groups underwent formalinfixation, paraffin embedding, and slice preparation. Then cell apoptosis in tumor tissues was evaluated by TUNEL staining using a commercial Kit (Roche, Mannheim, Germany) following the producer's guideline. Image was photographed under a fluorescence microscope (Olympus, Tokyo, Japan).

\section{Immunohistochemistry (IHC)}

The sections were obtained as described in Tunel assay. After deparaffinization and dehydration, the sections were treated with citrate buffer ( $\mathrm{pH}$ 6.0), followed by the heat pretreatment at $80{ }^{\circ} \mathrm{C}$ and blocking with endogenous peroxide. Next, the sections were incubated with SCARA5 and Ki67 antibodies, respectively, followed by the incubation of second antibody. Ultimately, the sections were mounted with neutral resin and observed under a light microscope (Nikon, ECLIPSE CI).

\section{Statistical analysis}

Quantitative data produced from at least biological repeats were analyzed for statistical significance with the SPSS 20.0 software. The Student $t$ test and ANOVA were performed to evaluate differences between two and $>2$ 
groups, respectively. A p value of $<0.05$ was set as the threshold for significant differences.

\section{Results}

\section{SCRAR5 expression was down-regulated in CRC cells}

Both the results of qPCR and western blotting showed obviously lower mRNA and protein levels of SCRAR5 in CRC cell lines (SW620, SW480, HT29, and HCT116) than that in normal HCoEpic cell line (Fig. 1A, B). Notably, SW480 and HT29 cell line, which had the lowest and highest expression of SCRAR5, respectively, were used for the further experiments.

\section{Effect of SCRAR5 abnormal expression on CRC cells}

SW480 cells were transfected with OE-SCRAR5, OE-NC, shSCRAR5, and shNC, respectively. Compared with cells with OE-NC or shNC, the mRNA level of SCRAR5 was remarkably up-regulated in cells transfected with OESCRAR5, while SCRAR5 expression was significantly down-regulated in cells with shSCRAR5 ( $\mathrm{p}<0.05$, Fig. 2A). Cell proliferation assays revealed that compared with control and OE-NC or siNC groups, SCRAR5 overexpression obviously inhibited cell viability in time-dependent manner ( $<<0.05$, Fig. 2B), decreased the EdU fluorescence (Fig. 2C), and reduced the clone number (Fig. 2D); reversely, SCRAR5 knockdown increased cell viability (Fig. 2B), the EdU fluorescence (Fig. 2C), and the clone number (Fig. 2D). In addition, SCRAR5 overexpression induced cell apoptosis, while SCRAR5 knockdown suppressed cell apoptosis

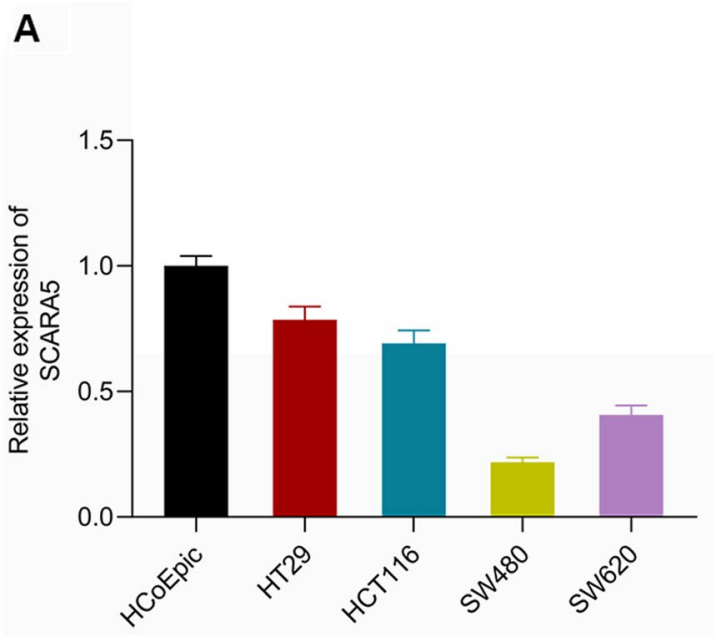

Fig. 1 Scavenger receptor class A member 5 (SCRAR5) expression in colorectal cancer (CRC) cells. The mRNA and protein levels of SCRAR5 in CRC cell lines (HT29, HCT116, SW480, and SW620) when compared with control cells (Fig. 2E). Meanwhile, cell migration was also evaluated by wound healing assay and transwell assay. Compared with control and OE-NC or siNC groups, the cell migration rate was decreased and the relative wound area was increased in cells transfected with OE-SCRAR5, while which were reversed in cells with shSCRAR5 (p < 0.05, Fig. 2F, G). Furthermore, HT29 cells were transfected with shSCRAR5, and shNC, respectively. Compared with cells with shNC, SCRAR5 expression was significantly down-regulated in cells with shSCRAR5 ( $p<0.05$, Fig. 3A). Cell proliferation assays revealed that compared with control and siNC groups, SCRAR5 knockdown increased cell viability (Fig. 3B), the EdU fluorescence (Fig. 3C), and the clone number (Fig. 3D). Additionally, SCRAR5 knockdown suppressed cell apoptosis when compared with control cells (Fig. 3E). Compared with control and siNC groups, the cell migration rate was increased and the relative wound area was decreased in cells transfected with shSCRAR5 ( $p<0.05$, Fig. 3F, G).

\section{Effect of SCRAR5 abnormal expression on PI3K/AKT/ mTOR pathway in CRC cells}

Furthermore, PI3K/AKT/mTOR pathway was detected to reveal the mechanism of SCRAR5 overexpression that inhibited the growth and migration of CRC cells. Compared with cells with OE-NC or shNC, the protein level of SCRAR5 was highly expressed in cells transfected with OESCRAR5, while lowly expressed in cells with shSCRAR5 (Fig. 4). Interestingly, SCRAR5 overexpression decreased the expression of p-PI3K, p-AKT, and p-mTOR, while SCRAR5 knockdown promoted their expressions (Fig. 4).

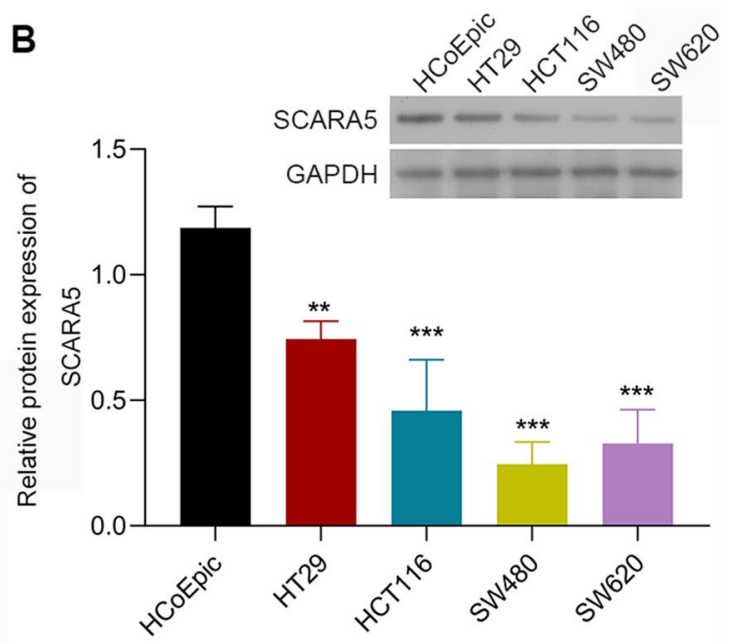

was significantly lower than that in normal HCoEpic cell line by qPCR (A) and western blotting (B), respectively 


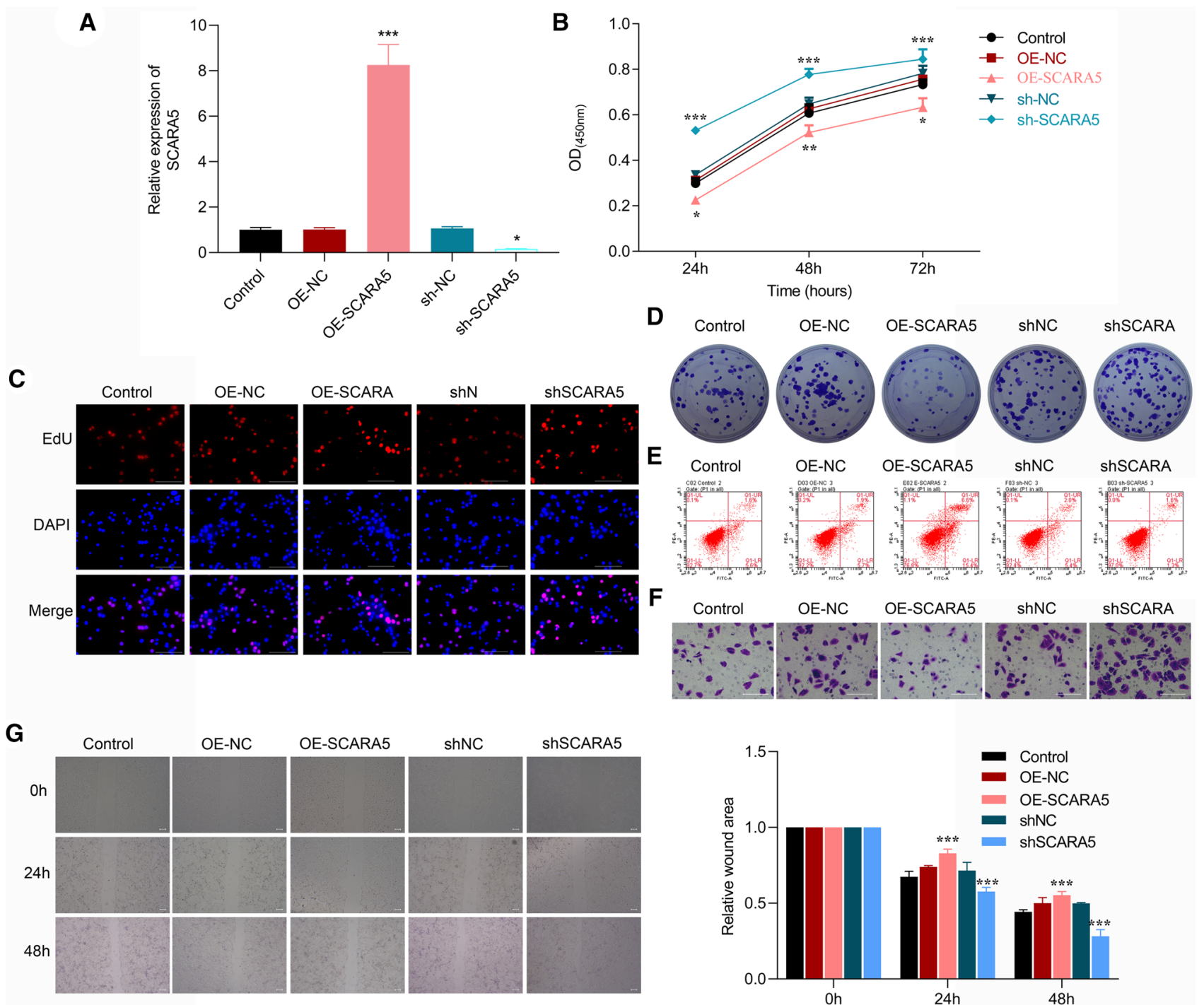

Fig. 2 Scavenger receptor class A member 5 (SCRAR5) overexpression inhibited cell proliferation, induced cell apoptosis, and suppressed cell migration in SW480 cells. A The mRNA level of SCRAR5 in SW480 cells transfected with SCRAR5 overexpression plasmid (OE-SCRAR5), SCRAR5 silence plasmid (shSCRAR5), and the corresponding negative controls $\mathrm{OE}-\mathrm{NC}$, and shNC, respectively. B Cell viability of SW480 cells in control, OE-NC, OE-SCRAR5, shSCRAR5, and shNC groups, respectively, in time-dependent manner by CCK-8 assay. C Cell proliferation of SW480 cells in control, OE-NC, OE-SCRAR5, shSCRAR5, and shNC groups, respectively, by5-ethynyl-2'- deoxyuridine (EdU) assay. D The clone number

\section{In vivo anti-tumor effect of SCRAR5 overexpression on CRC xenograft mice}

In vivo experiments found that the tumor volumes of CRC xenograft mice were reduced in the OE-SCRAR5 group in comparison to the control or OE-NC group in a time-dependent manner $(\mathrm{p}<0.01$, Fig. 5A, B), while and the tumor volume was increased in the shSCRAR5 group $(\mathrm{p}<0.01$, of SW480 cells in control, OE-NC, OE-SCRAR5, shSCRAR5, and shNC groups, respectively, by colony-forming assay. E Cell apoptosis of SW480 cells in control, OE-NC, OE-SCRAR5, shSCRAR5, and shNC groups, respectively, by annexin V-fluorescein isothiocyanate (FITC)/propidium iodide (PI) double-staining assay. F The cell migration of SW480 cells in control, OE-NC, OE-SCRAR5, shSCRAR5, and shNC groups, respectively, by Transwell assay. G The relative wound area of SW480 cells in control, OE-NC, OESCRAR5, shSCRAR5, and shNC groups, respectively, by wound healing assay

Fig. 5A, B). Meanwhile, both the mRNA and protein levels of SCRAR5 were elevated after OE-SCRAR5 treatment and decreased after shSCRAR5 treatment in mice (Fig. 5C, D). Furthermore, Tunel assay revealed the increased apoptotic cells in the OE-SCRAR5 group and reduced apoptotic cells in the shSCRAR5 group compared with the control and NC groups (Fig. 5E). IHC showed that the Ki67 expression trend in various groups was consistent with the tumor volume of 
A

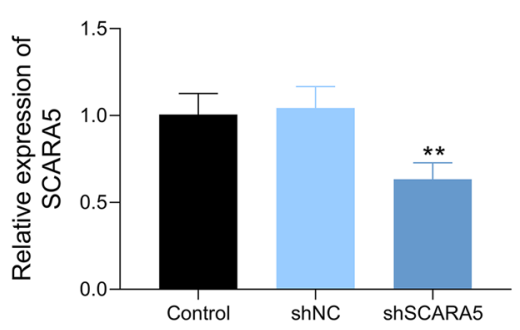

C
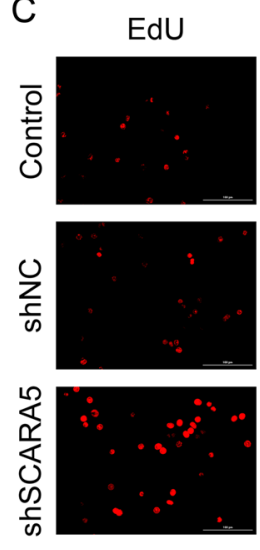

D

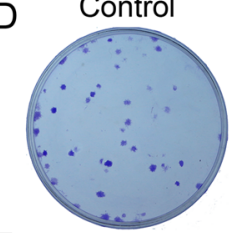

E

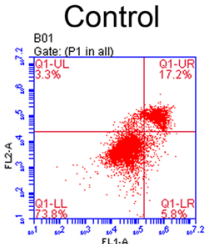

shNC

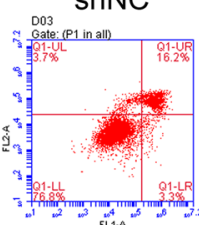

F Control

shNC

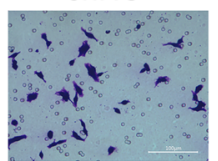

G Control

shNC

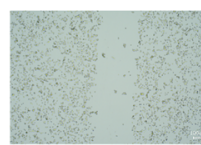

守

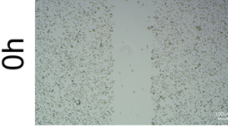

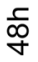

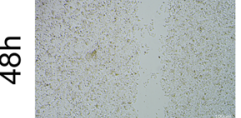

B
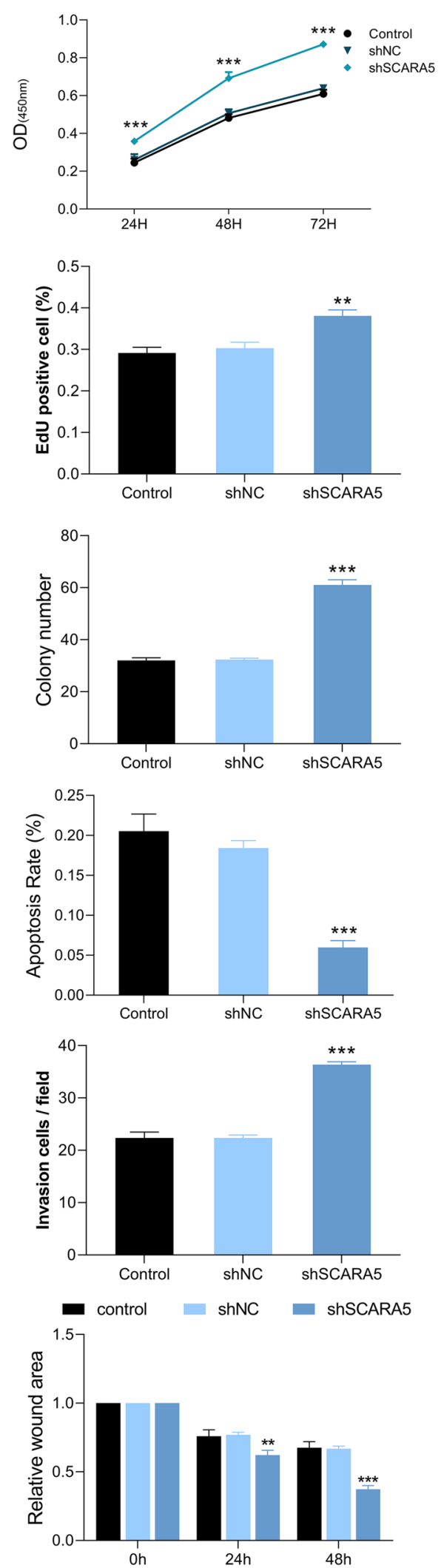
4Fig. 3 Scavenger receptor class A member 5 (SCRAR5) silence promoted cell proliferation, inhibited cell apoptosis, and increased cell migration in HT29 cells. A The mRNA level of SCRAR5 in HT29 cells transfected with SCRAR5 silence plasmid (shSCRAR5), and the corresponding negative control shNC, respectively. B Cell viability of HT29 cells in control, shSCRAR5, and shNC groups, respectively, in time-dependent manner by CCK-8 assay. C Cell proliferation of HT29 cells in control, shSCRAR5, and shNC groups, respectively, by5-ethynyl-2'- deoxyuridine (EdU) assay. D The clone number of HT29 cells in control, shSCRAR5, and shNC groups, respectively, by colony-forming assay. E Cell apoptosis of HT29 cells in control, shSCRAR5, and shNC groups, respectively, by annexin V-fluorescein isothiocyanate (FITC)/propidium iodide (PI) double-staining assay. F The cell migration of HT29 cells in control, shSCRAR5, and shNC groups, respectively, by Transwell assay. $\mathbf{G}$ The relative wound area of HT29 cells in control, shSCRAR5, and shNC groups, respectively, by wound healing assay

mice (Fig. 5F). SCRAR5 expression detected by IHC also similar to the $\mathrm{qPCR}$ and western blotting assays in various groups (Fig. 5G).

\section{Discussion}

High metastasis and drug resistance to chemotherapeutic drugs have become a key factor hindering the long-term survival of patients with CRC. The abnormal expression of SCRAR5 has been reported to be involved in various cancers. However, the roles and mechanisms of SCARA5 during the occurrence and progression CRC still unclear. In the present study, we found the down-regulation of SCRAR5 in CRC cells. Importantly, SCRAR5 overexpression obviously inhibited CRC cell growth and migration, which might be related to PI3K/AKT/mTOR pathway. Consistently, in vivo experiments also revealed that SCRAR5 overexpression exerted anti-tumor effects in CRC xenograft mice.

Several studies have investigated the role of SCRAR5 in cancers, and they have shown that SCRAR5 participates in cancer progression as tumor suppressor. For example, SCRAR5 is reported to be dramatically down-regulated in breast cancer, and promote breast carcinogenesis by regulating promoter methylation (Ulker et al. 2018). Also, SCRAR5 is proposed to be lowly expressed in breast cancer, and SCRAR5 overexpression restrains the tumor proliferation and metastasis by serving as tumor suppressor (You et al. 2017). Liu et al. have shown that down-regulation of SCRAR5 aggravates hepatocellular carcinoma progression via regulating $\beta$-catenin degradation in vitro and in vivo experiments (Liu et al. 2018b). Notably, SCRAR5 is reported to involve with CRC progression (Liu et al. 2020). Similarly, this study found that SCRAR5 was down-regulated in CRC cells. Thus, we prompted that SCRAR5 might be a potential tumor suppressor in CRC.

To further uncover the role of SCRAR5 in CRC, we evaluated the role of SCRAR5 abnormal expression on cell proliferation, apoptosis, and migration in CRC cells as well as in CRC xenograft mice, and the results revealed that SCARA5 upregulation remarkedly inhibited cell growth in SW480 cells, as well as inhibited tumor growth in CRC xenograft mice, which indicated that SCARA5 participated in abnormal tumor proliferation in CRC in vitro and in vivo. Cell apoptosis is key indicator in cancer research, which can reflect the cancer progression and prognosis (Xu et al. 2019; Xue et al. 2019). Both in vitro and in vivo experiments in this study revealed SCARA5 overexpression induced cell apoptosis, thereby inhibiting tumor growth. The migration ability of tumor cells is another pivotal indicator to evaluate cell aggressiveness and tumor progression. A previous report has shown that SCARA5 knockdown contributes to epithelial-to-mesenchymal transition-induced migration in lung carcinoma A549 cells (Liu et al. 2013). The current study also found that overexpression of SCARA5 exhibits inhibiting effect on cell migration ability of SW480 cells. These
Fig. 4 Scavenger receptor class A member 5 (SCRAR5) overexpression inhibited PI3K/ AKT/mTOR pathway in SW480 cells. The protein expression of SCRAR5, phosphorylated-phosphatidylinositol-3-hydroxykinase (p-PI3K), PI3K, p-AKT, AKT, p-mTOR, and mTOR of SW480 cells in control, OE-NC, OE-SCRAR5, shSCRAR5, and shNC groups, respectively, by western blotting
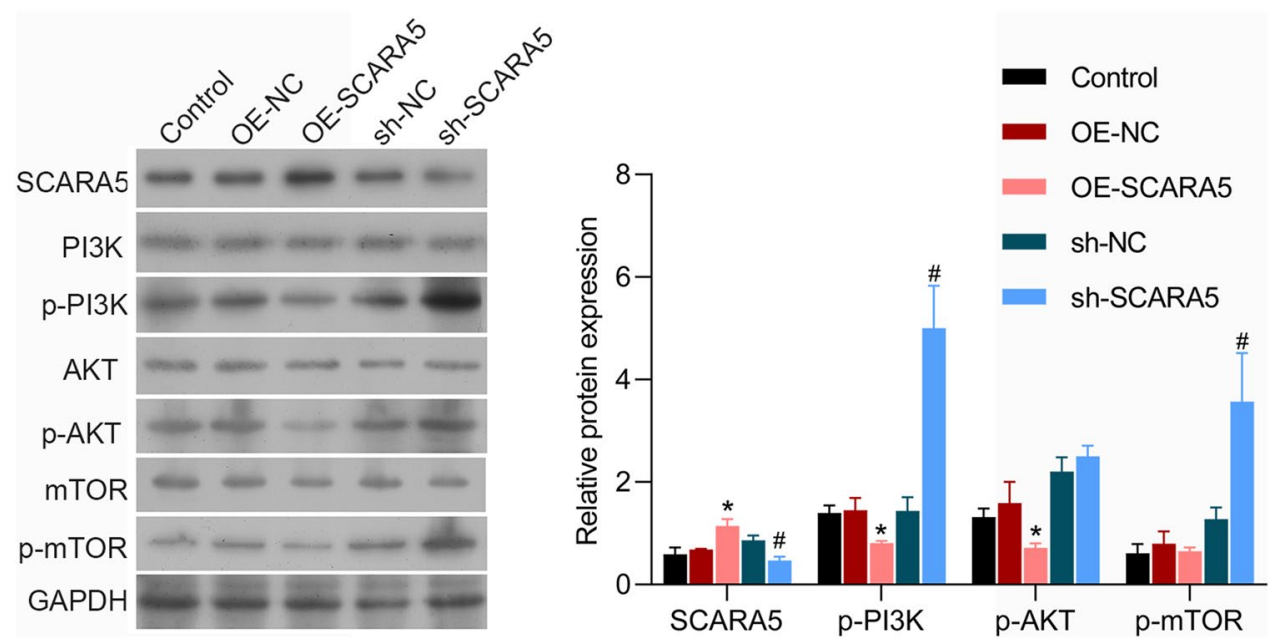


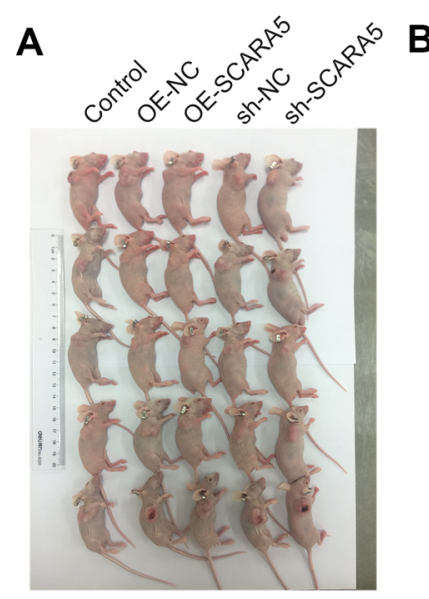

C

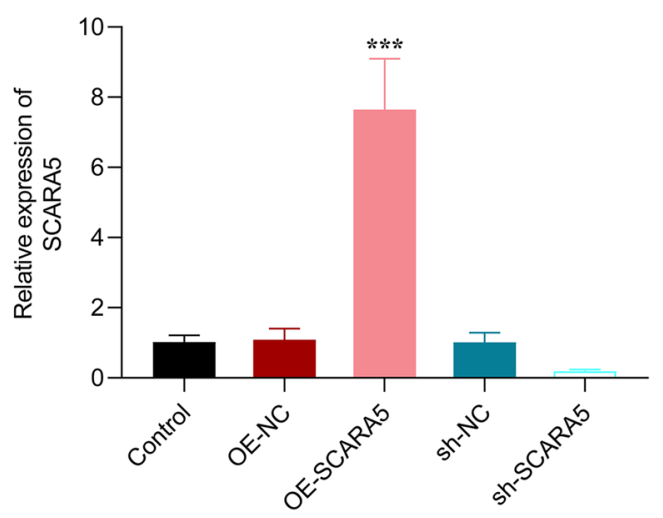

E

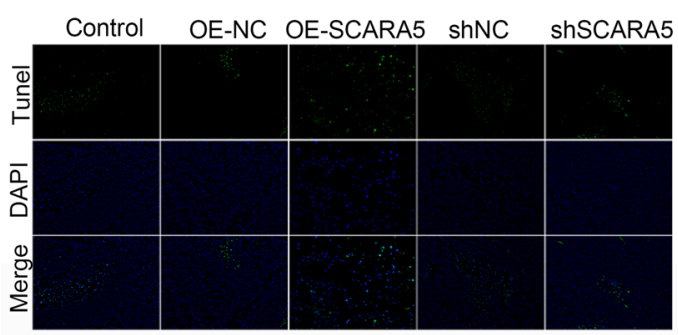

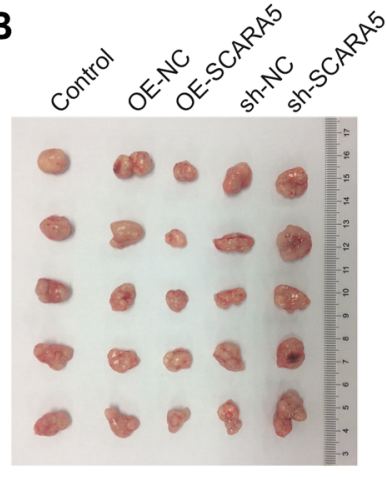
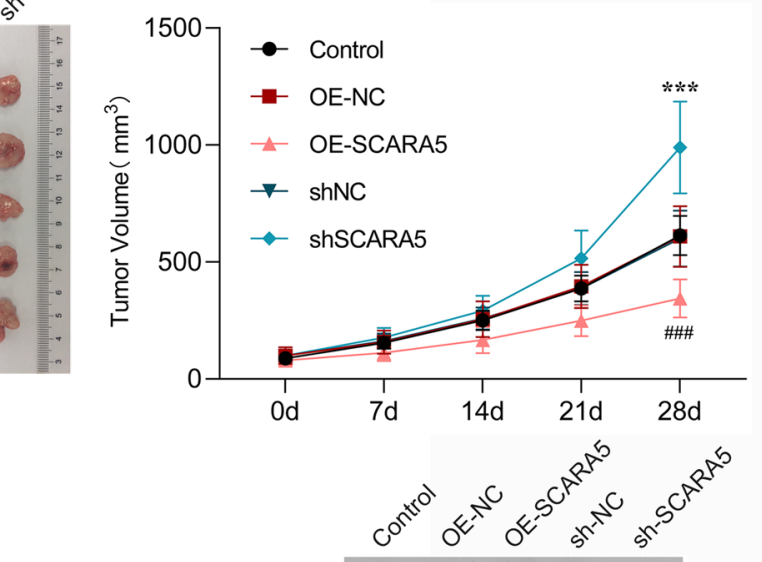

D
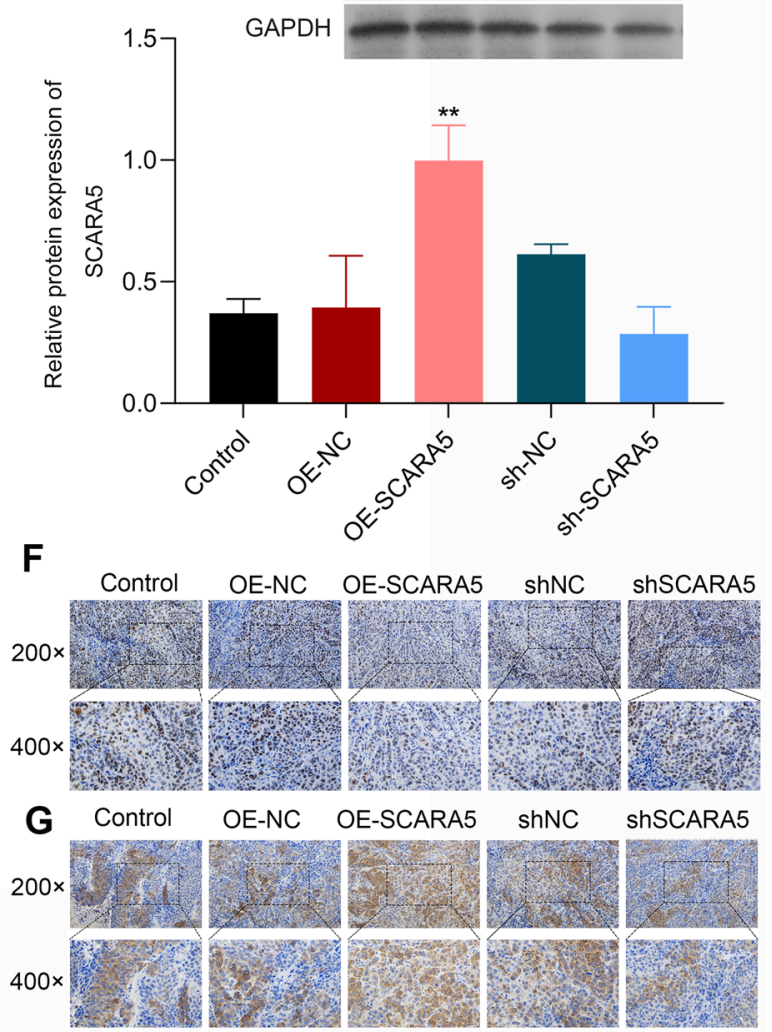

Fig. 5 Scavenger receptor class A member 5 (SCRAR5) overexpression inhibited tumor growth in colorectal cancer (CRC) xenograft mice. A The tumor image of CRC xenograft mice in control, OE-NC, OE-SCRAR5, shSCRAR5, and shNC groups. B Tumor volumes of CRC xenograft mice in control, OE-NC, OE-SCRAR5, shSCRAR5, and shNC groups in a time-dependent manner. The mRNA $(\mathbf{C})$ and protein (D) levels of SCRAR5 of CRC xenograft mice in control,

OE-NC, OE-SCRAR5, shSCRAR5, and shNC groups. E The cell apoptosis of CRC xenograft mice in control, OE-NC, OE-SCRAR5, shSCRAR5, and shNC groups by Tunel assay. The Ki-67 (F) and SCRAR5 (G) expressions of CRC xenograft mice in control, OE-NC, OE-SCRAR5, shSCRAR5, and shNC groups by immunohistochemistry

influences of SCARA5 abnormal expression in PI3K/AKT/ mTOR pathway. PI3K/AKT pathway has been reported to be closely implicated in cell proliferation, apoptosis and migration (Noorolyai et al. 2019). The phosphorylation of PI3K is 
able to modify the protein structure of AKT and then activate AKT, which further regulates tumor cell proliferation, differentiation, apoptosis, and migration (Noorolyai et al. 2019). In addition, mTOR served as a downstream target of PI3K/AKT pathway also can be activated to involve with tumor cell progression and chemoresistance (Polivka Jr and Janku 2014). Interestingly, previous study has demonstrated that activation of PI3K/AKT/mTOR signaling pathway is beneficial to cell proliferation and migration in CRC (Narayanankutty 2019; Pandurangan 2013). Additionally, PI3K/ $\mathrm{Akt} / \mathrm{mTOR}$ pathway is involved in the metastatic initiation and drug resistance events of CRCs (Narayanankutty 2019). Due to the pivotal role of PI3K/Akt/mTOR pathway in CRC, more researches have focused on the natural and synthetic small molecules that can effectively target this pathway as potent inhibitors of CRCs (Bahrami et al. 2018). Fortunately, data from several clinical trials confirm the therapeutic effects of pathway inhibitors such as BYL719, KRX-0401, MK-2206, BEZ235, and everolimus in CRC patients (Bahrami et al. 2018). Consistently, our study also revealed that SCARA5 overexpression down-regulated the expression of p-PI3K, p-AKT, and p-mTOR, while SCRAR5 silence contributed to the activation of PI3K/AKT/mTOR pathway. Therefore, we speculated that PI3K/AKT/mTOR signaling pathway might participate in the effects of SCRAR5 on cell proliferation, apoptosis and migration in CRC.

In conclusion, the data of this study suggested that SCRAR5 was a potential tumor suppressor by regulating tumor growth and metastasis in CRC, which might be associated with PI3K/AKT/mTOR signaling pathway.

\section{Acknowledgements None.}

Author contributions YL, YQX, FP, XYT and JW designed the study, YL, PF, XYT and JW performed the experiments and statistical analysis. YL, YQX and FP drafted and revised the manuscript. All authors read and approved the final manuscript.

\section{Declarations}

Conflict of interest Yi Li, Feng Peng, Xiangyun Tan, Jin Wang, and Yeqing $\mathrm{Xu}$ declare that they have no conflict of interest.

Ethical approval All research protocols and animal experiments in this study were reviewed and approved by Experimental Animal Welfare Ethics Committee of Central South University.

Open Access This article is licensed under a Creative Commons Attribution 4.0 International License, which permits use, sharing, adaptation, distribution and reproduction in any medium or format, as long as you give appropriate credit to the original author(s) and the source, provide a link to the Creative Commons licence, and indicate if changes were made. The images or other third party material in this article are included in the article's Creative Commons licence, unless indicated otherwise in a credit line to the material. If material is not included in the article's Creative Commons licence and your intended use is not permitted by statutory regulation or exceeds the permitted use, you will need to obtain permission directly from the copyright holder. To view a copy of this licence, visit http://creativecommons.org/licenses/by/4.0/.

\section{References}

Bahrami A, Khazaei M, Hasanzadeh M, ShahidSales S, Joudi Mashhad M, Farazestanian M, Sadeghnia HR, Rezayi M, Maftouh M, Hassanian SM (2018) Therapeutic potential of targeting PI3K/AKT pathway in treatment of colorectal cancer: rational and progress. J Cell Biochem 119:2460-2469

Canton J, Neculai D, Grinstein S (2013) Scavenger receptors in homeostasis and immunity. Nat Rev Immunol 13:621-634

Ersahin T, Tuncbag N, Cetin-Atalay R (2015) The PI3K/AKT/mTOR interactive pathway. Mol Biosyst 11:1946-1954

Gusev EY, Zotova NV, Zhuravleva YA, Chereshnev VA (2020) Physiological and pathogenic role of scavenger receptors in humans. Med Immunol 22:7-48

Keum N, Giovannucci E (2019) Global burden of colorectal cancer: emerging trends, risk factors and prevention strategies. Nat Rev Gastroenterol Hepatol 16:713-732

Liu J, Hu G, Chen D, Gong A, Soori G, Dobleman T, Chen X-M (2013) Suppression of SCARA5 by Snail1 is essential for EMTassociated cell migration of A549 cells. Oncogenesis 2:e73-e73

Liu H, Hu J, Pan H, Luo D, Huang M, Xu W (2018a) CSN5 promotes hepatocellular carcinoma progression by SCARA5 inhibition through suppressing $\beta$-catenin ubiquitination. Dig Dis Sci 63:155-165

Liu H, Hu J, Wei R, Zhou L, Pan H, Zhu H, Huang M, Luo J, Xu W (2018b) SPAG5 promotes hepatocellular carcinoma progression by downregulating SCARA5 through modifying $\beta$-catenin degradation. J Exp Clin Cancer Res 37:229

Liu Y, Yr T, Sun Ww Ju, Wt FuY, Lz W, Li J, Cp Z, Zy Z, Lp Z (2018c) Identification of SCARA5 as a potential biomarker for oral squamous cell carcinoma using MALDI-TOF-MS analysis. Proteom Clin Appl 12:1700180

Liu J, Zeng M, Shi P, Cao Y, Zhang J, Xie Y (2020) SCARA5 is a novel biomarker in colorectal cancer by comprehensive analysis. Clin Lab 66(7). https://doi.org/10.7754/Clin.Lab.2019.191015

Mamoor S (2020) The scavenger receptor class A member 5, SCARA5, is differentially expressed in the brain metastases of patients with metastatic breast cancer. OSF Preprints. https:// doi.org/10.31219/osf.io/9t6ru

Mármol I, Sánchez-de-Diego C, Pradilla Dieste A, Cerrada E, Rodriguez Yoldi MJ (2017) Colorectal carcinoma: a general overview and future perspectives in colorectal cancer. Int J Mol Sci 18:197

Narayanankutty A (2019) PI3K/Akt/mTOR pathway as a therapeutic target for colorectal cancer: a review of preclinical and clinical evidence. Curr Drug Targets 20:1217-1226

Noorolyai S, Shajari N, Baghbani E, Sadreddini S, Baradaran B (2019) The relation between PI3K/AKT signalling pathway and cancer. Gene 698:120-128

Pandurangan AK (2013) Potential targets for prevention of colorectal cancer: a focus on PI3K/Akt/mTOR and Wnt pathways. Asian Pac J Cancer Prev 14:2201-2205

Plüddemann A, Neyen C, Gordon S (2007) Macrophage scavenger receptors and host-derived ligands. Methods 43:207-217

Polivka J Jr, Janku F (2014) Molecular targets for cancer therapy in the PI3K/AKT/mTOR pathway. Pharmacol Ther 142:164-175

Porta C, Paglino C, Mosca A (2014) Targeting PI3K/Akt/mTOR signaling in cancer. Front Oncol 4:64 
PrabhuDas MR, Baldwin CL, Bollyky PL, Bowdish DM, Drickamer K, Febbraio M, Herz J, Kobzik L, Krieger M, Loike J (2017) A consensus definitive classification of scavenger receptors and their roles in health and disease. J Immunol 198:3775-3789

Rejhová A, Opattová A, Čumová A, Slíva D, Vodička P (2018) Natural compounds and combination therapy in colorectal cancer treatment. Eur J Med Chem 144:582-594

Shukla HD (2017) Comprehensive analysis of cancer-proteogenome to identify biomarkers for the early diagnosis and prognosis of cancer. Proteomes 5:28

Siegel RL, Miller KD, Goding Sauer A, Fedewa SA, Butterly LF, Anderson JC, Cercek A, Smith RA, Jemal A (2020) Colorectal cancer statistics, 2020. CA Cancer J Clin 70:145-164

Ulker D, Ersoy YE, Gucin Z, Muslumanoglu M, Buyru N (2018) Downregulation of SCARA5 may contribute to breast cancer via promoter hypermethylation. Gene 673:102-106

Wang S, Zhou D, Xu Z, Song J, Qian X, Lv X, Luan J (2019) Antitumor drug targets analysis: current insight and future prospect. Curr Drug Targets 20:1180-1202

Whelan FJ, Meehan CJ, Golding GB, McConkey BJ, Bowdish DM (2012) The evolution of the class A scavenger receptors. BMC Evol Biol 12:1-11

Xu Z, Yu Z, Tan Q, Wei C, Tang Q, Wang L, Hong Y (2019) MiR876-5p regulates gastric cancer cell proliferation, apoptosis and migration through targeting WNT5A and MITF. Biosci Rep 39:BSR20190066. https://doi.org/10.1042/BSR20190066
Xue J, Jin X, Wan X, Yin X, Fang M, Liu T, Zhao S (2019) Effects and mechanism of tanshinone II A in proliferation, apoptosis, and migration of human colon cancer cells. Med Sci Monit 25:4793-4800

Yan N, Zhang S, Yang Y, Cheng L, Li C, Dai L, Dai L, Zhang X, Fan P, Tian H (2012) Therapeutic upregulation of Class A scavenger receptor member 5 inhibits tumor growth and metastasis. Cancer Sci 103:1631-1639

You K, Su F, Liu L, Lv X, Zhang J, Zhang Y, Liu B (2017) SCARA5 plays a critical role in the progression and metastasis of breast cancer by inactivating the ERK1/2, STAT3, and AKT signaling pathways. Mol Cell Biochem 435:47-58

Yu B, Cheng C, Wu Y, Guo L, Kong D, Zhang Z, Wang Y, Zheng E, Liu Y, He Y (2020a) Interactions of ferritin with scavenger receptor class A members. J Biol Chem 295:15727-15741

Yu G, Gong X, Fu A, Hou Y, Xia J, Wang N, Zhao W, Hu H, Ouyang J, Fei L (2020b) Aberrant expression of SCARA5 in lung adenocarcinoma and its clinical significance. Res Sq. https://doi.org/10. 21203/rs.3.rs-117152/v1

Publisher's Note Springer Nature remains neutral with regard to jurisdictional claims in published maps and institutional affiliations. 